\title{
Expression of ADAMTS13 in human endometrium and its potential role in recurrent pregnancy loss
}

\section{Type}

Research paper

\section{Keywords}

endometrium, recurrent pregnancy loss, expression, vWF, ADAMTS13, decidua

\begin{abstract}
Introduction

The mechanisms underlying the pathogenesis of recurrent pregnancy loss (RPL) and the effective approaches to treat this disease still remain vague and absent. Proteinases of ADAMTS family play important roles in embryonic growth and development. Our previous study suggest a role of ADAMTS13 during pregnancy. Current Study was to determine the expression of ADAMTS13 in human endometrium and its association with RPL.
\end{abstract}

Material and methods

The spatiotemporal expression of ADAMTS13 in human endometrium was examined by immunohistochemistry. real-time PCR sand western blot were then employed to determine the mRNA and protein expression levels of ADAMTS13 in human endometrium. Proteolytic cleavage of FRETS-VWF73 were performed to determine the activity of ADAMTS13 in plasma and that secreted by human endometrium. ELISA was carried out to measure plasma VWF antigen.

\section{Results}

We show that proteolytically active ADAMTS13 is expressed in human endometrium throughout the menstrual cycle and pregnancy. The decidual expression levels of mRNA and protein in women with RPL were significantly lower compared with women with uncomplicated pregnancies $(P<0.01$, $\mathrm{P}<0.05$, respectively). Furthermore, significantly reduced plasma ADAMTS13 activity (median [range] 69.09 [65.2-93.7]\% versus $93.62[88.1-115.6] \%, P<0.001)$ and elevated plasma VWF antigen levels (median [range] of 125.5 [54.2-262.8]\% versus $91.9[80.4-138.7] \%, P<0.01$ ) were detected in RPL patients compared with the control group.

\section{Conclusions}

These findings suggest that ADAMTS13 may play a role in embryo implantation and the pathogenesis of recurrent pregnancy loss. Further investigation on ADAMTS13 gene knockout animal models is necessary for understanding the molecular mechanisms of the biological roles of ADAMTS13 during gestation. 
Expression of ADAMTS13 in human endometrium and its potential role in recurrent pregnancy loss

Expression of ADAMTS13 in human endometrium 


\section{Abstract}

Introduction The mechanisms underlying the pathogenesis of recurrent pregnancy loss (RPL) and the effective approaches to treat this disease still remain vague and absent. Proteinases of ADAMTS family play important roles in embryonic growth and development. Our previous study suggest a role of ADAMTS13 during pregnancy. Current Study was to determine the expression of ADAMTS13 in human endometrium and its association with RPL.

Material and methods The spatiotemporal expression of ADAMTS13 in human endometrium was examined by immunohistochemistry. real-time PCR and western blot were then employed to determine the mRNA and protein expression levels of ADAMTS13 in human endometrium. Proteolytic cleavage of FRETS-VWF73 were performed to determine the activity of ADAMTS13 in plasma and that secreted by human endometrium. ELISA was carried out to measure plasma VWF antigen.

Results We show that proteolytically active ADAMTS13 is expressed in human endometrium throughout the menstrual cycle and pregnancy. The decidual expression levels of mRNA and protein in women with RPL were significantly lower compared with women with uncomplicated pregnancies $(\mathrm{P}<0.01, \mathrm{P}<0.05$, respectively). Furthermore, significantly reduced plasma ADAMTS13 activity (median [range] $69.09[65.2-93.7] \%$ versus $93.62[88.1-115.6] \%, \mathrm{P}<0.001)$ and elevated plasma VWF antigen levels (median [range] of 125.5 [54.2-262.8]\% versus $91.9[80.4-138.7] \%, \mathrm{P}<0.01$ ) were detected in RPL patients compared with the control group.

Conclusions These findings suggest that ADAMTS13 may play a role in embryo implantation and the pathogenesis of recurrent pregnancy loss. Further investigation on ADAMTS13 gene knockout animal models is necessary for understanding the molecular mechanisms of the biological roles of ADAMTS13 
during gestation.

Key words ADAMTS13; VWF; expression; endometrium; decidua; recurrent pregnancy loss

\section{Introduction}

Recurrent pregnancy loss (RPL) currently refers to two or more consecutive spontaneous losses until 20 weeks of gestation, which frustrates $1-5 \%$ of childbearing couples(1). Possible causes of RPL include genetic defects, anatomical abnormalities, immune dysfunctions, infections, endocrine disturbances, thrombotic disorders and unexplained causes(1-3). Although some couples have beneficiated from targeted interventions, the specific mechanisms underlying the pathogenesis of RPL as well as the effective treatments still remain vague and absent.

Degradation of the extracellular matrix (ECM) and angiogenesis in the human endometrium are crucial processes necessary for the morphological and functional changes of the endometrium during menstrual cycle and pregnancy $(4,5)$. As a novel family of secreted metalloproteinases, the ADAMTS (A Disintegrin And Metalloproteinase with Thrombospondin repeats) composes of more than 20 genetically distinct subtypes in humans(6). Accumulating evidence converges to suggest that these novel proteinases play important roles in embryonic growth and development(7), and the cyclic remodeling events that take place in reproductive tissues(8). Vascular endothelial growth factor (VEGF) is believed to play a key role in angiogenesis in the endometrium(4). However, as abnormal angiogenesis and aberrant distribution or expression of ECM components in the endometrial stroma have been related to infertility and pregnancy loss (9), it is necessary to determine the full repertoire of proteinases expressed in the endometrium, their distribution and regulation, and, ultimately, their individual role(s) in the development of a uterine environment that is capable of sustaining pregnancy. 
ADAMTS13 is mainly synthesized in the liver(10), and its primary function is to cleave von Willebrand factor (VWF) in circulation, anchored on the endothelial surface, and at the sites of vascular injury. Severe deficiency of plasma ADAMTS13 activity resulting from ADAMTS13 gene mutations or autoantibodies against ADAMTS13 leads to hereditary or acquired (idiopathic) Thrombotic Thrombocytopenic Purpura (TTP)(11, 12). Recently, ADAMTS13 have been detected in a broad spectrum of human cells including endothelial cells, megakaryocytes, platelets, renal tubular epithelial cells, glomerular endothelial cells and glomerular podocytes at various levels(13) We have previously shown that ADAMTS13 is expressed in human placental tissue, as well as in placental explant and trophoblast cell cultures, and that it can promote angiogenesis and trophoblastic cell development(14). Significantly reduced levels of placental ADAMTS13 expression was detected in patients with preeclampsia(14). These findings suggest a role of ADAMTS13 during pregnancy. However, the expression of ADAMTS13 in the human endometrium has not ever been reported. The change of plasma ADAMTS13 activity and plasma VWF antigen level in RPL patients are rarely reported. Therefore, our aim was to determine the expression of ADAMTS13 in the endometrium and its association with RPL.

\section{Material and Methods}

\section{Tissue collection}

All study patients were recruited from the The First Affiliated Hospital of Zhengzhou University, Zhengzhou, Henan, China, between January 2018 and January 2019. This study was reviewed and approved by the Ethics Committee of The First Affiliated Hospital of Zhengzhou University, and informed consent was obtained from all participants. The endometrial specimens (10 proliferative; 10 secretory) were obtained from premenopausal women undergoing surgery for non-endometrial pathology, 
such as leiomyomata or benign ovarian cysts. Data from only those tissues that were found to be normal by microscopic examination are reported here. An experienced pathologist determined the endometrial dating according to the criteria of Noyes et al(15). Decidual tissues and plasma were obtained from 30 patients undergoing early pregnancy terminations for psychosocial reasons and 25 unexplained RPL patients undergoing therapeutic abortions of early gestations. RPL was defined as the loss of two or more consecutive pregnancies before the 20 weeks of gestation(1). Ectopic pregnancy, hydatidiform mole, or biochemical pregnancy were excluded from their pregnancy histories in clinical assessment. All RPL patients underwent karyotype analysis of aborted embryo. RPL patients with abnormal karyotype, uterine abnormalities, endocrine abnormalities, or immunologic dysfunctions were excluded. The third trimester decidual tissues $(n=10)$ from normal pregnancies were picked up from the gauze used to scrub the uterus immediately after cesarean section.

In the present study we exclusively used fresh endometrial and decidual tissue samples; any histological signs of tissue damage such as necrosis, old blood clots or infections were excluded from the study. One part was fixed in $4 \%$ buffered formalin for subsequent paraffin embedding and the major part was either snap-frozen in liquid nitrogen and stored at $-80^{\circ} \mathrm{C}$ for RNA and protein extraction or used immediately for explant culture. Prior to RNA/ protein isolation, tissue samples were cut into sections on a cryostat. One representative section of each sample was stained with haematoxylin to define representative tissues without signs of necrosis. Tissue was selected to obtain approximately equal amounts of stromal tissue, glands and other structures. For decidual fragments, another section was stained immunohistochemically with HLA-G to identify extravillous trophoblast. Only those decidual samples with invasive trophoblasts and no signs of necrosis were chosen for RNA and protein extraction. 
Normal liver tissue was obtained from the resected livers of patients with liver cancer prior to chemotherapy and/or radiotherapy. Tissue was taken from areas unaffected by the cancer and determined normal by the pathologist.

\section{Antibodies.}

Polyclonal rabbit anti-human ADAMTS13 antibody (F481) was produced at the University of Alabama at Birmingham, Birmingham Alabama using recombinant human ADAMTS13 variant with CUB domain deleted (i.e. DelCUB) and recombinant murine full-length ADAMTS13 at 9:1 ratio (Open Biosystems, Huntsville, AL). Monoclonal mouse anti-human HLA-G antibody and monoclonal rabbit anti-human CD34 antibody were purchased from Abcam (Cambridge, UK). Isotype rabbit IgG or mouse IgG were purchased from Sigma (Aldrich, St. Louis, USA).

\section{Cell culture and Transient expression of ADAMTS13}

Chinese Hamster Ovary (CHO) cells were cultured in DMEM/F-12 containing 10\% fetal bovine serum (FBS, Gibco BRL, Carlsbad, CA, USA), $50 \mathrm{U} / \mathrm{ml}$ penicillin and $50 \mathrm{mg} / \mathrm{ml}$ streptomycin in a $5 \% \mathrm{CO}_{2}$ humidified incubator at $37^{\circ} \mathrm{C}$. Cells were seeded onto 6-well culture plates and grown to $95 \%$ confluence prior to transfection. Transfection was carried out with a plasmid encoding a full-length human ADAMTS13 mixed with Lipofectamine ${ }^{\mathrm{TM}} 3000$ (Invitrogen, Carlsbad, CA, USA). Cells were harvested after 48 hours transfection and stored at $-80^{\circ} \mathrm{C}$ until use.

Organ culture

Endometrial tissues and decidual tissues were prepared and cultured as previously described(16, 17). All 
dissection was performed in a laminar-flow hood where the tissue was rinsed twice in fresh medium

Decidual tissues were divided into decidua parietalis which was free of fetal tissues and decidua capsularis in which trophoblast cells were attached to the decidua, and the latter were used for culture.

Endometrial tissues and decidual tissues were minced into small pieces $\sim 2 \mathrm{~mm}^{3}$ and then plated to a density of $30-40 \%$ of the well in small pieces using 6-well culture plates. Explant culture proceeded in $3 \mathrm{~mL}$ of serum free DMEM/F-12 media, containing $2 \mathrm{mM}$ glutamine, $50 \mathrm{U} / \mathrm{ml}$ penicillin and $50 \mathrm{mg} / \mathrm{ml}$ streptomycin in a $5 \% \mathrm{CO}_{2}$ humidified incubator at $37^{\circ} \mathrm{C}$. After 24 hours of incubation, culture medium was collected, centrifuged and concentrated by using Amicon® Ultra Centrifugal $50 \mathrm{kDa}$ (Millipore, Bedford, MA). The supernatant was used for VWF-cleaving activity assay. The tissues were collected and fixed in $4 \%$ buffered formalin for subsequent paraffin embedding.

\section{RNA isolation and cDNA preparation}

Total RNA from endometrial tissues was prepared with the TRIzol® (Invitrogen, Carlsbad, CA, USA), according to the manufacturer's protocol. The cDNA was synthesized from total RNA using the reverse transcription kit (TaKaRa, Japan) under the following conditions: 5 min incubation at $65{ }^{\circ} \mathrm{C}$ for primer annealing, $37^{\circ} \mathrm{C}$ for 15 min for cDNA synthesis, and 5 min termination at $98^{\circ} \mathrm{C}$ and then stored at $-80{ }^{\circ} \mathrm{C}$ until use.

\section{Real-time PCR}

Quantitation of ADAMTS13 transcripts was performed by a real-time PCR (ABI prism 7000; Applied Biosystems) using the SYBR Premix ExTaq Kit (TaKaRa, Japan) and adjusted to the quantitative expression of glyceraldehyde-3-phosphate dehydrogenase (GAPDH) from the corresponding unknowns. 
The PCR primers used were as follows: ADAMTS13, forward , 5'-TGTG CTGC CCAA ATAC

CCTA-3'and reverse, 5'-AACG GGAA GCAA TCAC CAAC-3'; HLA-G, forward ,

5'-GAGGAGACACGGAACACCAAG-3' and reverse, 5'-GTCGCAGCCAATCATCCACT-3'; CD34,

forward , 5'-CTGCCTTCTGGGTTCATGAGT-3'and reverse,

5'-GGTGGTGAACACTGTGCTGATTAC-3'. GAPDH,

forward, 5'-AAGGTCATCCCTGAGCTGAAC-3'and reverse, 5'-ACGCCTGCTTCACCACCTTCT-3.

Normal liver RNA was used as positive control(10). Blank CHO cell RNA was used as a negative control

\section{Immunohistochemistry}

The endometrial samples were immediately fixed with $4 \%$ neutral paraformaldehyde overnight and then

embedded into paraffin wax. ADAMTS13 expression was determined by immunohistochemistry staining.

Briefly, paraffin-embedded endometrium sections $(5 \mathrm{~mm})$ were dewaxed in xylene, and then rehydrated through descending ethanol to PBS. $0.3 \%$ hydrogen peroxide in methanol was used to quench the endogenous peroxidase activity in the sample. Antigen retrieval was performed in preheated $\left(95-100^{\circ} \mathrm{C}\right)$ $0.05 \%$ sodium citrate buffer for 20 minutes. The sections were blocked with goat serum, and then incubated with primary antibodies against ADAMTS13 $(20 \mu \mathrm{g} / \mathrm{ml})$ or HLA-G $(10 \mu \mathrm{g} / \mathrm{ml})$ or CD34 $(10 \mu \mathrm{g} / \mathrm{ml})$ at $4^{\circ} \mathrm{C}$ overnight. After that, the slides were washed in PBS containing $0.5 \%$ Tween 20 (Sigma, Aldrich, St. Louis, MO, USA) before incubating with horseradish peroxidase-labeled secondary antibody (anti-rabbit and anti-mouse, respectively) in a humidor at room temperature for 1 hour. Peroxidase substrate 3, 30-diaminobenzidene tetrahydrochloride (Dako, USA) was used to visualize the specific antibody binding and this was monitored under a microscope (Olympus IX73, Tokyo, Japan) to ensure 
proper intensity. And finally, the sections were counterstained with hematoxylin. In the negative control experiments, primary antibodies were omitted or isotype rabbit IgG or mouse IgG (Sigma-Aldrich, St. Louis, MO, USA) was used. The immunostaining was repeated three times on sections from the same sample. Immunostaining scoring of ADAMTS13, HLA-G or CD34 in the sample was evaluated on the basis of staining intensity and positively stained areas by three independent observers as previously described (19).

\section{Western blotting}

Expressions of ADAMTS13 protein in endometrium tissues was also determined by Western blotting.

Total protein was prepared by lysing CHO cells, endometrial tissues with RIPA lysis buffer (Beyotiem, Shanghai, China) containing a protease inhibitor cocktail (Roche Applied Science, Indianapolis, Indian). Protein samples were separated by $8 \%$ sodium dodecyl sulphate-polyacrylamide gel electrophoresis (SDS-PAGE) under reducing conditions and transferred to a polyvinylidene fluoride membrane (Millipore, Billerica, MA). The membranes were incubated sequentially with primary antibodies against ADAMTS13 $(2 \mu \mathrm{g} / \mathrm{ml})$ and then HRP-conjugated secondary antibody. Proteins were detected by an enhanced chemiluminescent (ECL) detection kit (Amersham, Biosciences, Uppsala, Sweden), and the image was captured using VisionWorksLS image acquisition and analysis software and the EC3 imaging system (UVP LLC, Upland, CA). Lysate from CHO cells transiently transfected with ADAMTS13 plasmid was used as a positive control.

\section{Determination of ADAMTS13 activity}

The fluorigenic substrate, FRETS-VWF73, was purchased from Peptides International (Louisville, KY, 
USA) and applied for the determination of ADAMTS13 enzyme activity according to the protocol provided by the supplier with minor modifications (20). Briefly, the test citrated plasma were diluted 1:20 in assay buffer( $5 \mathrm{mM}$ Bis-Tris, $25 \mathrm{mM} \mathrm{CaCl} 2,0.005 \%$ Tween $20, \mathrm{pH} 6.0$ ), While the culture medium samples were diluted 1:1 with the assay buffer. Then the diluted samples were mixed with $5 \mu \mathrm{M}$ FRETS-VWF73 substrate solution $(20 \mu \mathrm{l}$ each) in white 384 -well plates. Fluorescence was measured at $37^{\circ} \mathrm{C}$ every $2 \mathrm{~min}$ for $1 \mathrm{~h}$ in Chameleon microplate reader (Hidex, Turku, Finland) equipped with a 340 $\mathrm{nm}$ excitation and a $460 \mathrm{~nm}$ emission filter. The reaction rate was calculated by linear regression analysis of fluorescence over time. A two-fold dilution series of normal human plasma (mixed from citrated plasma samples of 10 healthy blood donors) was applied as standard curve, 100\% ADAMTS13 activity was set at the reaction rate observed in the 1:20 diluted plasma sample and the1:1 diluted culture media sample. The intra-assay coefficient of variation (CV) was $<5 \%$, the inter-assay CV was $6-9 \%$ (measured at 60 and $100 \%$ activity levels).

\section{Determination of plasma VWF antigen levels}

VWF antigen (VWF:Ag) levels were measured in citrated plasma by an enzyme-linked immunosorbent assay (ELISA) using commercially available antibodies (Dakopatts, Glostrup, Denmark). Microtiter plates (Immunoplate Maxisorb, Nunc, Roskilde, Denmark) were coated with polyclonal rabbit anti-human VWF antibody diluted 1:800 in sodium bicarbonate buffer, $\mathrm{pH}$ 9.6. After overnight incubation at $4{ }^{\circ} \mathrm{C}$, plates were washed in Tris-buffered saline containing $0.05 \%$ Tween 20 (TBS-T). The plates were incubated with plasma samples diluted 1:500 or standard control plasma (dilution range 1:250 to 1:8,000) for $2 \mathrm{~h}$ at room temperature. After washes with TBS-T, horse radish peroxidase (HRP)-conjugated polyclonal rabbit anti-human VWF antibody was added to the plates and incubated for $1 \mathrm{~h}$, followed by 
addition of ortho-phenylenediamine (OPD). The optical density was measured at $492 \mathrm{~nm}$. Results are expressed as percentages of a standard composed of pooled human plasma of 10 healthy blood donors.

\section{Statistical analysis}

The bands from Western blotting was quantified by MetaView Image Analyzing System (Version 4.50; Universal Imaging Co., Downingtown, PA, USA) and normalized in respect to the corresponding fragment concentration of the housekeeping genes GAPDH. Normal distribution of data was assessed by the Shapiro-Wilk test. The t-test were performed using the Statistical Package for Social Science (SPSS for Windows package release 20.0; SPSS Inc., Chicago, IL, USA) and indicated in Results and Figure legends. All data were expressed as the mean \pm SEM. Statistical significance was set as $\mathrm{p}<0.05$.

\section{Results}

\section{Characteristics of patients}

The baseline characteristics of the 2 study groups are presented in the Table 1. Controls were matched to RPL cases by maternal age and incidence of smoking. Number of previous pregnancies and pregnancy losses were significantly higher in the RPL group than those in control group.

ADAMTS13 mRNA is expressed in human endometrium throughout the menstrual cycle and pregnancy

ADAMTS13 mRNA was detected by real-time PCR in human endometrium throughout the menstrual cycle and pregnancy. Our previous study showed predominant expression of ADAMTS13 in extravillous trophoblast and endothelial cells. We run for HLA-G probe to evaluate the level of extravillous 
trophoblast in decidua tissues by real-time PCR. Human liver that is known to express ADAMTS13 (10) was positive, while the blank $\mathrm{CHO}$ cell showed no signal in the real-time PCR, suggesting that the amplified ADAMTS13 mRNA signals in the endometrial tissues were specific. GAPDH was used to normalize the total RNA added during the amplification. The results were expressed as the ratio of ADAMTS13 or HLA-G to GAPDH (Fig. 1A,B).

ADAMTS13 protein is synthesized by human endometrium throughout the menstrual cycle and pregnancy Immunoblots with anti-human ADAMTS13 IgG showed that ADAMTS13 proteins of $120 \mathrm{kDa}$ and 190 $\mathrm{kDa}$ in the lysates of endometrial tissues during the menstrual cycle and pregnancy (Fig. 1C). No bands were detected in the blank $\mathrm{CHO}$ cell lysates as a negative control.

Localization of ADAMTS13 in human endometrium throughout the menstrual cycle and pregnancy

The cellular localization of ADAMTS13 in human endometrium throughout the menstrual cycle and pregnancy was determined by immunohistochemistry. The results showed that ADAMTS13 protein was detected mainly in stroma cells, vascular endothelial cells, and some glandular epithelial cells in proliferative endometrium and secretory endometrium; ADAMTS13-positive staining was also detected in extravillous trophoblasts in decidua from first trimester and third trimester. HLA-G was used as a marker of extravillous trophoblasts and CD34 a marker of endothelial cells in the decidua. Tissue sections stained with isotype IgG or only stained with secondary antibody showed negative staining (Fig. 2). 


\section{the menstrual cycle and pregnancy}

Explant culture medium of endometrial tissues throughout the menstrual cycle and pregnancy were harvested and concentrated. Immunohistochemistry was performed to evaluate the expression of HLA-G and CD34 in cultured decidual tissues (Fig. 3A). Results showed that HLA-G and CD34 expression levels in 1 st trimester decidua were significantly lower than those in 3rd trimester decidua (Fig. 3B,3C). real-time PCR showed that expression level of HLA-G mRNA was obviously lower in 1st trimester decidua than that in 3rd trimester decidua(Fig. 3D). FRETS-VWF73 substrate was used to determine the proteolytic activity as described in the Materials and Methods. The ADAMTS13 activity in the conditioned medium of these explants was 4.61\% (range 2.6-6.4\%), 5.6\% (range 3.4-8.1\%), 4.82\% (range $2-7.4 \%$ ) and 5.88\% (range 3.9-7.8\%), respectively (Fig. 3E).

\section{Plasma ADAMTS13 activity are reduced and VWF antigen levels are increased in RPL patients}

There was significant difference in plasma ADAMTS13 activity between the RPL group and the control group (median [range] 69.09 [65.2-93.7]\% versus 93.62 [88.1-115.6]\%, $\mathrm{P}<0.001$ ) (Fig. 3F). Furthermore plasma VWF antigen levels were significantly higher in RPL patients than in control women (median [range] of 125.5 [54.2-262.8]\% versus 91.9[80.4-138.7]\%, P < 0.01) (Fig. 3G).

\section{ADAMTS13 mRNA and protein expression are reduced in deciduae of RPL patients}

Immunostaining of the deciduae did not reveal difference in the ADAMTS13 expression pattern on extravillous trophoblasts between RPL patients and controls (Fig. 4Aa,b). ADAMTS13 (Fig. 4B) and HLA-G (Fig. 4E)expression levels in RPL decidua were significantly decreased compared with that in 
control decidua, while CD34 (Fig. 4H) expression level showed no obviously difference between the two group. The levels of ADAMTS13 mRNA in decidual tissues were determined by real-time PCR. As shown, decidual tissues had lower levels of ADAMTS13 mRNA in patients with RPL compared with those from normal pregnancy (Fig. 4C). This may be explained by the obviously reduced presence of extravillous trophoblast as indicted by the decreased expression of HLA-G mRNA in RPL tissue samples (Fig. 4D). In consistent with real-time PCR, immunoblot showed that obviously reduced levels of ADAMTS13 protein in the deciduae from RPL patients compared with those in the control (Fig. 4F and $4 G)$.

\section{Discussion}

The current study demonstrates for the first time that proteolytically active ADAMTS13 is present in human endometrium tissues throughout the menstrual cycle and pregnancy. What is more, ADAMTS13 expression is significantly reduced in the deciduae from RPL women. In addition, we firstly discovered the reduction of plasma ADAMTS13 activity and elevation of plasma VWF antigen level in RPL patients.

The presence of many members of the ADAMTS family have been demonstrated in human endometrial tissues(21-25), many albeit at low levels, indicating that these metalloproteinases may play important roles in implantation and placentation. We have previously demonstrated that proteolytically active ADAMTS13 is expressed in human placenta throughout the pregnancy; in the placenta, ADAMTS13 is mainly expressed by trophoblast and fetal blood vessel endothelium; ADAMTS13 synthesis is significantly reduced in placental tissues from PE patients and that exposed to hypoxic conditions; what is more, recombinant full-length ADAMTS13 promotes proliferation, migration, invasion and network formation of trophoblasts(14). Taken together, these observations suggest important 
roles of ADAMTS13 in pregnancy and likely the pathogenesis of preeclampsia. Current study demonstrated for the first time the expression of ADAMTS13 mRNA and protein in human endometrium tissues throughout the menstrual cycle and pregnancy.

Other ADAMTS members, known as aggrecanases including ADAMTS-1, -4, -5, -8, -9 have also been detected in human deciduae(21, 23-25). Mice null-mutant for ADAMTS-1 or ADAMTS-5 subtype are capable of undergoing normal decidualization(26) or proceed to develop large endometrial cysts during the reproductive cycle(25), indicating an uterine environment capable of supporting pregnancy, therefore the role(s) of these subtypes in the process of the highly regulated, multimolecular complex structures present in the endometrial ECM under normal and pathological conditions remain to be elucidated. ADAMTS13 gene-knockout mice are viable and fertile(27, 28), suggesting that the establishment and maintenance of pregnancy is not dependent upon the regulated expression of ADAMTS13 in the placenta or decidua and that other local ADAMTS members may play overlapping/compensatory roles in this multi-step reproductive process. It is usually the case to see such redundancy in gene families(29).

While the well-established biological role of ADAMTS13 is to regulate the process of hemostasis and thrombosis, ADAMTS13 may also involve in inflammation, angiogenesis and tissue remodeling. Apart from the typical matrix proteins of ECM, such as fibronectin, laminin, and fibrincollagen, fibrin and fibrinogen are found deposited in fibrosis tissue, indicating that coagulation factors may also be constituent of ECM. VWF is the only known substrate of ADAMTS13. While VWF is secreted by endothelial cells in arteries into both the lumen and the subendothelial space(30), ADAMTS13, as a metalloprotease, has been speculated to be related to ECM degradation. VWF is found upregulated in the 
microvascular endothelial cells in the area of fiber-like structure and necrosis during liver injury(31). Therefore, it is reasonable to presume that ADAMTS13 released at the time of tissue injury may prevent excessive ECM constituent deposition(32) and development of fibrosis in the liver(33). Likewise, ADAMTS13 expressed by podocytes and tubular cells may possibly involved in tissue remodeling in $\operatorname{situ}(34,35)$.

There has been an increased interest in the role of ADAMTS13 in angiogenesis. Recently, Lee et al have demonstrated that ADAMTS13 might exert either pro-angiogenic or anti-angiogenic effects depending on the cellular environments(36). In the absence of excessive VEGF, ADAMTS13 promotes angiogenesis by phosphorylation of $\operatorname{VEGFR2}$ and upregulating $\operatorname{VEGF}(36,37)$. In the presence of excessive VEGF, ADAMTS13 appears to inhibit angiogenesis depending on the C-terminal TSP1 repeats, by competing with VEGF for binding to its receptor VEGFR. Collectively, ADAMTS13 expressed in deciduae has the potential to contribute to the development of uterine environment capable of supporting a pregnancy via the degradation of the decidual ECM and/or the extensive vascular changes that occur in this dynamic tissue during implantation and placentation.

RPL is a complicated problem related to chromosomal abnormalities, autoimmune disorders, endocrine dysfunction, infectious processes, environmental toxins, advanced maternal and paternal age, and congenital or structural uterine anomalies $(1,38)$. However, until now none of them have provided a clear explanation about its etiology and pathogenesis. Invasion of extravillous trophoblast, vascular remodeling, and decidualization are the prominent processes for a successful embryo implantation(39). Decidualization involves the reorganization of the decidual ECM. Angiogenesis is a fundamental process occurring during embryonic development and reproductive cycles(40). Deficiency in decidualization and 
angiogenesis due to abnormal protein expression involved in this process might lead to the aberrant growth of the fetus or severely, the pregnancy loss. Interestingly, in current study both the mRNA and protein expression level of ADAMTS13 revealed lower in decidua samples of RPL patients than those of normal control groups. This suggests that decidua ADAMTS13 may play a significant role in the modulation of the uterine environment, preparing the uterus for implantation of the developing conceptus and formation of a functional placenta during the establishment of pregnancy. However, further investigation on ADAMTS13 gene knockout animal models is necessary for understanding the molecular mechanisms of the biological roles of ADAMTS13 during gestation.

Maternal thrombophilia has been suggested as an important risk factor for RPL $(1,38)$. In this study, the activity of ADAMTS13 was found to be decreased RPL patients when compared with controls. The presence of increased amounts of VWF in RPL patients can probably be ascribed to the decrease levels of ADAMTS13 activity. Owing to the decrease of ADAMTS13 activity, ultralarge VWF multimers, which are biologically more active, circulate in RPL patients and this may lead to maternal thrombophilia. These data indicate that reduction in the levels of ADAMTS13 may play a role in the onset of RPL. Our previous study showed predominant expression of ADAMTS13 in extravillous trophoblast and endothelial cells and low or undetectable ADAMTS13 in nearby decidual stromal cells. Therefore, we speculate that the lower expression of ADAMTS13 in tissue extract and supernatant is a reflection of impaired trophoblast invasion and reduced presence of extravillous cytotrophoblast in RPL tissue sample, which was demonstrated by the decreased immunohistochemical staining intensity of EVT in RPL decidual tissue.

\section{Conclusion}


In summary, we determined the expression of ADAMTS13 in human endometrium tissues and that

ADAMTS13 expression is significantly reduced in the deciduae from RPL patients. In addition, we demonstrated the decrease of plasma ADAMTS13 activity and increase of plasma VWF antigen level in RPL patients. Collectively, these findings serve as the basis for future studies on the expression and function(s) of ADAMTS13 in the human endometrium under normal and pathological conditions.

Funding: The study was supported by the grants from Joint project of Medical science and Technology Research Program of Henan Province (LHGJ20190114 to Yun Feng), National Science Foundation of China (No. 31670844 to Ruixia Guo); Centaline thousand talents plan-centaline famous doctors (ZYQR201810107 to Ruixia Guo) and Program for Science and Technology Innovation Teams in Henan Province (2018JR0004 to Ruixia Guo).

\section{References}

1. Toth B, Würfel W, Bohlmann M, et al. Recurrent Miscarriage: Diagnostic and Therapeutic Procedures. Guideline of the DGGG, OEGGG and SGGG (S2k-Level, AWMF Registry Number 015/050). Geburtshilfe Frauenheilkd. 2018;78(4):364-381.

2. Mohammad Seyedhassani S, Houshmand M, Mehdi Kalantar S, et al. BAX pro-apoptotic gene alterations in repeated pregnancy loss. Arch Med Sci. 2011;7(1):117-122.

3. Deshmukh H, Way SS. Immunological Basis for Recurrent Fetal Loss and Pregnancy Complications. Annu Rev Pathol. 2019;14:185-210.

4. He X, Chen Q. Reduced expressions of connexin 43 and VEGF in the first-trimester tissues from women with recurrent pregnancy loss. Reprod Biol Endocrinol. 2016;14(1):46.

5. Benkhalifa M, Zayani Y, Bach V, et al. Does the dysregulation of matrix metalloproteinases contribute to recurrent implantation failure? Expert Rev Proteomics. 2018;15(4):311-323.

6. Fontanil T, Mohamedi Y, Cobo T, et al. Novel Associations Within the Tumor Microenvironment: Fibulins Meet ADAMTSs. Front Oncol. 2019;9(796). 
7. Zhong S, Khalil RA. A Disintegrin and Metalloproteinase (ADAM) and ADAM with thrombospondin motifs (ADAMTS) family in vascular biology and disease. Biochem Pharmacol. 2019;164:188-204.

8. Russell DL, Brown HM, Dunning KR. ADAMTS proteases in fertility. Matrix Biol. 2015;46:54-63.

9. Jokimaa V, Oksjoki S, Kujari $\mathrm{H}$, et al. Altered expression of genes involved in the production and degradation of endometrial extracellular matrix in patients with unexplained infertility and recurrent miscarriages. Mol Hum Reprod. 2002;8(12):1111-1116.

10. Uemura M, Tatsumi K, Matsumoto M, et al. Localization of ADAMTS13 to the stellate cells of human liver. Blood. 2005;106(3):922-924.

11. Mazepa MA, Park YA, Raval JS. Taking Empiricism out of Immune Thrombotic Thrombocytopenic Purpura: Current and Future Treatment Strategies. Transfus Med Rev. 2019;33(4):248-255.

12. Chiasakul T, Cuker A. Clinical and laboratory diagnosis of TTP: an integrated approach. Hematology Am Soc Hematol Educ Program. 2018;30(1):530-538.

13. Feng Y, Li X, Xiao J, et al. ADAMTS13: more than a regulator of thrombosis. Int $\mathbf{J}$ Hematol. 2016;104(5):534-539.

14. Xiao J, Feng Y, Li X, et al. Expression of ADAMTS13 in Normal and Abnormal Placentae and Its Potential Role in Angiogenesis and Placenta Development. Arterioscler Thromb Vasc Biol. 2017;37(9):1748-1756.

15. Noyes RW, Hertig AT, Rock J. Dating the endometrial biopsy. American journal of obstetrics and gynecology. 1975;122(2):262-263.

16. Wu WX, Glasier A, Norman J, et al. The effects of the antiprogestin mifepristone, in vivo, and progesterone in vitro on prolactin production by the human decidua in early pregnancy. Hum Reprod. $1990 ; 5(5): 627-631$.

17. Maslar IA, Ansbacher R. Effects of progesterone on decidual prolactin production by organ cultures of human endometrium. Endocrinology. 1986;118(5):2102-2108.

18. Turner N, Nolasco L, Tao Z, et al. Human endothelial cells synthesize and release ADAMTS-13. J Thromb Haemost. 2006;4(6):1396-1404.

19. Su S, Liu Q, Chen J, et al. A positive feedback loop between mesenchymal-like cancer cells and 
macrophages is essential to breast cancer metastasis. Cancer Cell. 2014;25(5):605-620.

20. Kokame K, Nobe Y, Kokubo Y, et al. FRETS-VWF73, a first fluorogenic substrate for ADAMTS13 assay. Br J Haematol. 2005;129(1):93-100.

21. Zhu H, Leung PC, MacCalman CD. Expression of ADAMTS-5/implantin in human decidual stromal cells: regulatory effects of cytokines. Hum Reprod. 2007;22(1):63-74.

22. Ng YH, Zhu H, Pallen CJ, et al. Differential effects of interleukin-1beta and transforming growth factor-beta1 on the expression of the inflammation-associated protein, ADAMTS-1, in human decidual stromal cells in vitro. Hum Reprod. 2006;21(8):1990-1999.

23. Wen J, Zhu H, Murakami S, et al. Regulation of A Disintegrin And Metalloproteinase with ThromboSpondin repeats-1 expression in human endometrial stromal cells by gonadal steroids involves progestins, androgens, and estrogens. The Journal of clinical endocrinology and metabolism. 2006;91(12):4825-4835.

24. Wen J, Zhu H, Leung PC. Gonadal steroids regulate the expression of aggrecanases in human endometrial stromal cells in vitro. J Cell Mol Med. 2013;17(10):1325-1334.

25. Shindo T, Kurihara H, Kuno K, et al. ADAMTS-1: a metalloproteinase-disintegrin essential for normal growth, fertility, and organ morphology and function. J Clin Invest. 2000;105(10):1345-1352.

26. Mittaz L, Russell DL, Wilson T, et al. Adamts-1 is essential for the development and function of the urogenital system. Biol Reprod. 2004;70(4):1096-1105.

27. Banno F, Kokame K, Okuda T, et al. Complete deficiency in ADAMTS13 is prothrombotic, but it alone is not sufficient to cause thrombotic thrombocytopenic purpura. Blood. 2006;107(8):3161-3166.

28. Motto DG, Chauhan AK, Zhu G, et al. Shigatoxin triggers thrombotic thrombocytopenic purpura in genetically susceptible ADAMTS13-deficient mice. J Clin Invest. 2005;115(10):2752-2761.

29. Madan P, Bridges PJ, Komar CM, et al. Expression of messenger RNA for ADAMTS subtypes changes in the periovulatory follicle after the gonadotropin surge and during luteal development and regression in cattle. Biol Reprod. 2003;69(5):1506-1514.

30. Ruggeri ZM. The role of von Willebrand factor in thrombus formation. Thromb Res. 2007;120(1):9.

31. Peng Y, Chen Q, Yang T, Tao Y, et al. Cultured mycelium Cordyceps sinensis protects liver sinusoidal endothelial cells in acute liver injured mice. Mol Biol Rep. 2014;41(3):1815-1827. 
32. Stanton H, Melrose J, Little CB, et al. Proteoglycan degradation by the ADAMTS family of proteinases. Biochim Biophys Acta. 2011;12(29):2.

33. Kim TH, Mars WM, Stolz DB, et al Expression and activation of pro-MMP-2 and pro-MMP-9 during rat liver regeneration. Hepatology. 2000;31(1):75-82.

34. Manea M, Tati R, Karlsson J, et al. Biologically active ADAMTS13 is expressed in renal tubular epithelial cells. Pediatr Nephrol. 2010;25(1):87-96.

35. Manea M, Kristoffersson A, Schneppenheim R, et al. Podocytes express ADAMTS13 in normal renal cortex and in patients with thrombotic thrombocytopenic purpura. $\mathrm{Br} \mathrm{J}$ Haematol. 2007;138(5):651-662.

36. Lee M, Rodansky ES, Smith JK, et al. ADAMTS13 promotes angiogenesis and modulates VEGF-induced angiogenesis. Microvasc Res. 2012;84(2):109-115.

37. Lee M, Keener J, Xiao J, et al. ADAMTS13 and its variants promote angiogenesis via upregulation of VEGF and VEGFR2. Cell Mol Life Sci. 2015;72(2):349-356.

38. Rai R, Regan L. Recurrent miscarriage. Lancet. 2006;368(9535):601-611.

39. Bhurke AS, Bagchi IC, Bagchi MK. Progesterone-Regulated Endometrial Factors Controlling Implantation. Am J Reprod Immunol. 2016;75(3):237-245.

40. Billhaq DH, Lee SH, Lee S. The potential function of endometrial-secreted factors for endometrium remodeling during the estrous cycle. Anim Sci J. 2020;91(1):7.

Fig. 1. ADAMTS13 activity was detected in the concentrated conditioned media of human endometrium throughout the menstrual cycle and pregnancy. A. ADAMTS13 mRNA is detected by real-time PCR in human endometrium throughout the menstrual cycle and pregnancy. Human liver mRNA was used as the positive control and blank CHO cell mRNA was used as the negative control. The data were standardized against the gene GAPDH. Pro, proliferative endometrium $(n=10)$; Sec, secretory endometrium $(n=10)$; 1st, first trimester decidua $(\mathrm{n}=10)$; 3rd, third trimester decidua $(\mathrm{n}=10)$; HL, human live. Results were presented as the mean \pm SEM. B. HLA-G mRNA was detected by real-time PCR in human decidual 
tissues. The data were standardized against the gene GAPDH. C. ADAMTS13 is synthesized by human endometrium throughout the menstrual cycle and pregnancy. Lysates from endometrium tissues exhibited bands at 190 and $120 \mathrm{kDa}$. A full-length recombinant ADAMTS13 (r-A13) secreted from CHO cells transiently transfected with an ADAMTS13 plasmid was used as a positive control and revealed a band at $190 \mathrm{kDa}$ under reducing conditions. No bands were detected in the CHO cell lysates as a negative control (data not shown).

Figure. 2. Expression of cellular ADAMTS13 in human endometrium throughout the menstrual cycle and pregnancy. Aa,d, ADAMTS13 expression in stroma cells (Str), vascular endothelial cells (indicated by arrowhead), and some glandular epithelial cells (Epi) in proliferative endometrium and secretory endometrium. Ba,d, ADAMTS13 expression in extravillous trophoblasts (EVT) in the decidua. Bb\&e, HLA-G was used as a marker of extravillous trophoblasts (EVT) in the decidua. Bc\&f, CD34 was used as a marker of blood vessel endothelium (End) in the decidua. Ab,e\&Bg,j. stained with isotype rabbit IgG. Bh,k. stained with isotype mouse IgG. Ac,f \& Bi,l. stained only with secondary antibody. Pro, proliferative endometrium; Sec, secretory endometrium; End, endovascular; 1st, first trimester decidua; 3rd, third trimester decidua. Bar $=50 \mu \mathrm{m}$.

Fig.3. ADAMTS13 activity was detected in the concentrated conditioned media of human endometrium throughout the menstrual cycle and pregnancy. Tissues after culture for preparation of conditioned media were immunohistologically evaluated. Aa,b. HLA-G was used as a marker of extravillous trophoblasts (EVT) in the decidua. Ac,d. CD34 was used as a marker of blood vessel endothelium (End) in the decidua. 
B, C. HLA-G or CD34 in the sample was evaluated on the basis of staining intensity and positively stained areas. HLA-G and CD34 expression levels in 1st trimester decidua were significantly lower than those in 3rd trimester decidua. * and $* *$ indicate $\mathrm{p}$ values of $<0.05$ and $<0.01$, respectively. D. Expression level of HLA-G mRNA was obviously lower in 1st trimester decidua than that in 3rd trimester decidua. The data were standardized against the gene GAPDH. * indicate $\mathrm{p}$ values of $<0.05$. E. The proteolytic activity of ADAMTS13 was determined by FRETS-VWF73 substrate. Tissue media from proliferative endometrium (Pro, $\mathrm{n}=10)$, secretory endometrium $(\mathrm{Sec}, \mathrm{n}=10)$, 1st trimester decidua $(1 \mathrm{st}, \mathrm{n}=10)$ and $3 \mathrm{rd}$ trimester decidua (3rd, $\mathrm{n}=10$ ) exhibited activity, with mean values of $4.61 \%$ (range $2.6-6.4 \%$ ), 5.6\% (range 3.4-8.1\%), 4.82\% (range $2-7.4 \%$ ) and 5.88\% (range 3.9-7.8\%) respectively. F. There was significant difference in plasma ADAMTS13 activity between the RPL $(n=25)$ and the control groups $(\mathrm{n}=30)$ (median [range] 69.09 [65.2-93.7]\% versus 93.62 [88.1-115.6]\%, P<0.001). G. Plasma VWF antigen levels were significantly higher in $\operatorname{RPL}(\mathrm{n}=25)$ women than in control women $(\mathrm{n}=30)$ (median [range] of $125.5[54.2-262.8] \%$ versus $91.9[80.4-138.7] \%, \mathrm{P}<0.01)$. ** and $* * *$ indicate $\mathrm{p}$ values of $<0.01$ and $<0.001$, respectively.

Fig.4. ADAMTS13 expression is down-regulated in RPL decidua. Aa,b. A13 staining on extravillous trophoblasts was present in a similar pattern in RPL and control groups. Human leukocyte antigen (HLA)-G was used for labeling for extravillous trophoblasts ADAMTS13 (Ac,d) and CD34 for blood vessel endothelium in decidua(Ae,f). ADAMTS13 (B) and HLA-G (E)expression levels in RPL decidua $(n=25)$ were significantly decreased compared with that in control decidua $(n=30)$, while CD34 $(H)$ expression level showed no obviously difference between the two group. .C, D. ADAMTS13 and HLA-G 
mRNA levels both showed significantly lower in RPL decidua $(n=25)$ than that in control decidua $(n=30)$.

F. Representative western-blot analysis that compared RPL $(n=25)$ versus control $(n=30)$ deciduae.

Quantification of the intensity of the bands is illustrated in G. Results were presented as the mean $\pm \mathrm{SEM}$.

$*$ and $* *$ indicate $\mathrm{p}$ values of $<0.05$ and $<0.01$, respectively. NS indicates no significant difference.

$\operatorname{Bar}=50 \mu \mathrm{m}$. 
Table 1 Clinical characteristics of the study sample.

\begin{tabular}{lccc}
\hline Characteristic & RPL $(\mathrm{n}=25)$ & Normal $(\mathrm{n}=30)$ & P value \\
\hline Maternal age (years) & $30.7 \pm 1.160$ & $29.7 \pm 0.904$ & 0.475 \\
Previous pregnancies & $3.5 \pm 0.203$ & $2.0 \pm 0.214$ & 0.000 \\
Pregnancy losses & $3.29 \pm 0.125$ & $0.44 \pm 0.166$ & 0.000 \\
Smoking & $2(8 \%)$ & $1(3.3 \%)$ & 0.585 \\
\hline
\end{tabular}

RPL, recurrent pregnancy loss. The data are shown as the mean \pm SEM or percentage. 
A

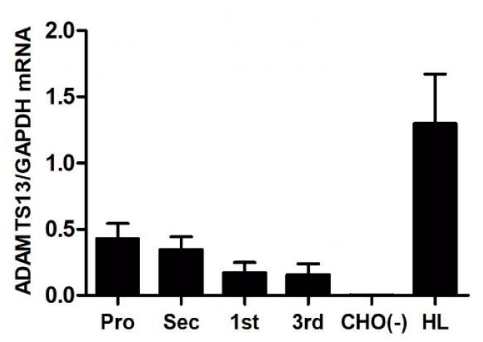

B

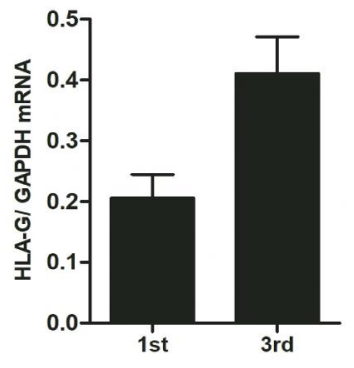

$\mathrm{C}$

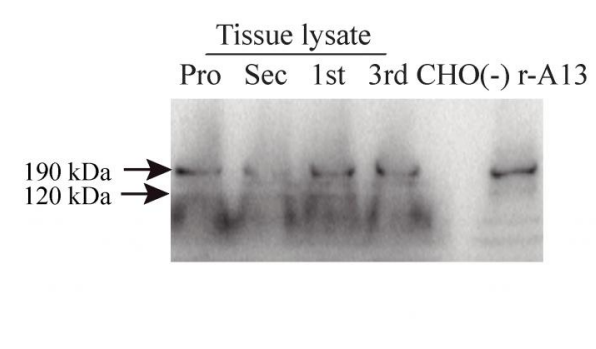

ADAMTS13 is detected in human endometrium throughout the menstrual cycle and pregnancy. 

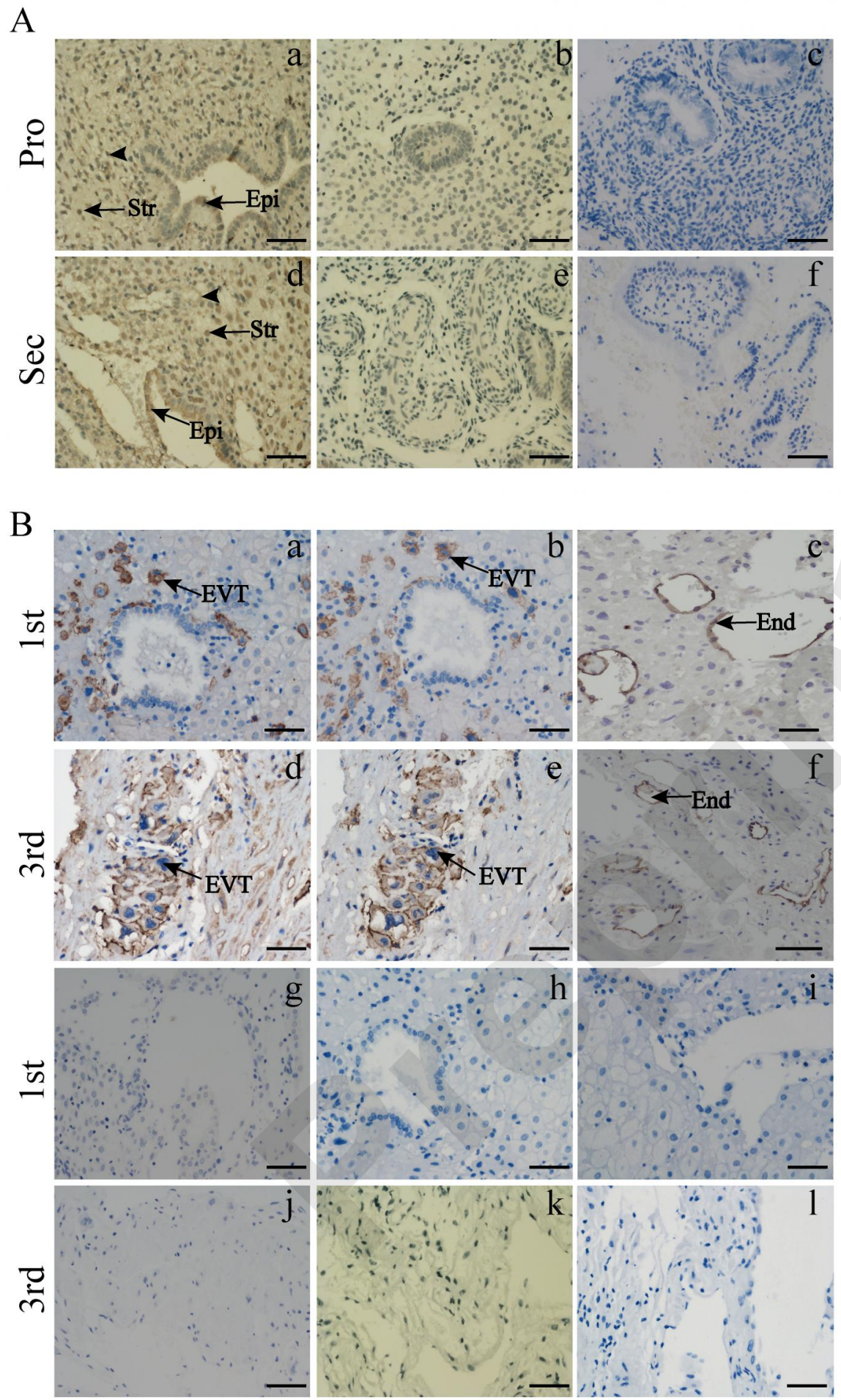

Expression of cellular ADAMTS13 in human endometrium throughout the menstrual cycle and pregnancy. 
A
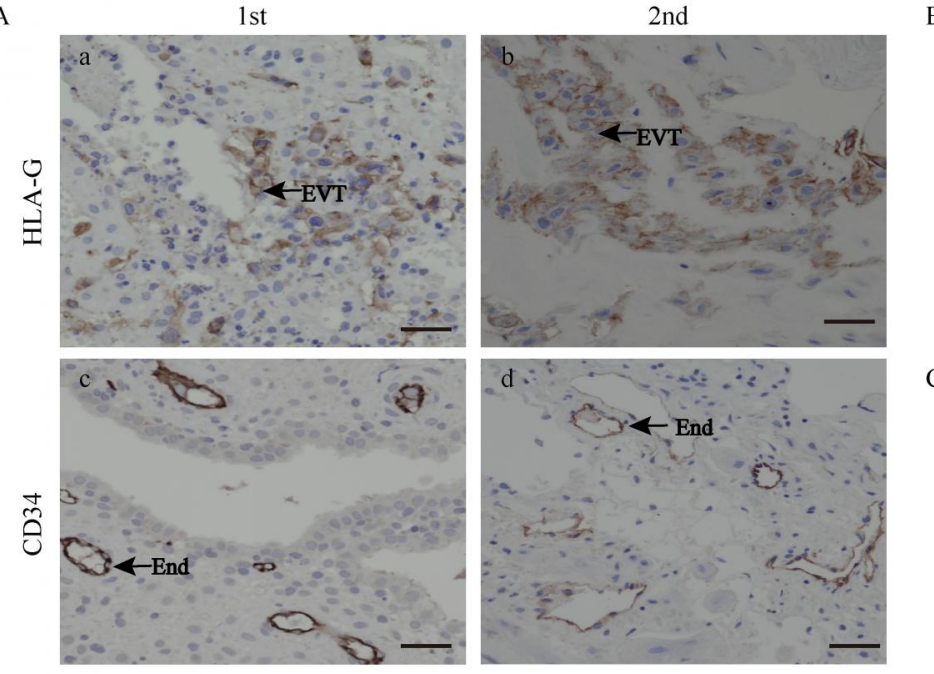

B

C
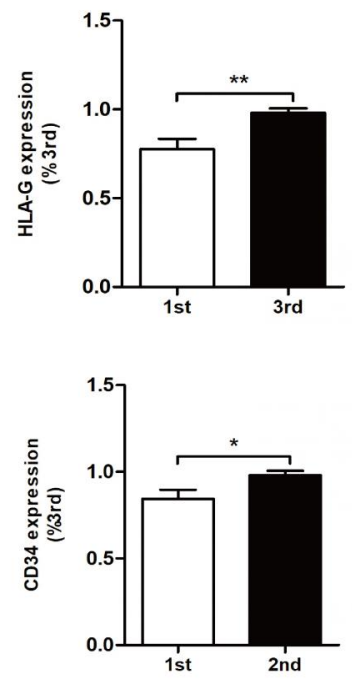

G

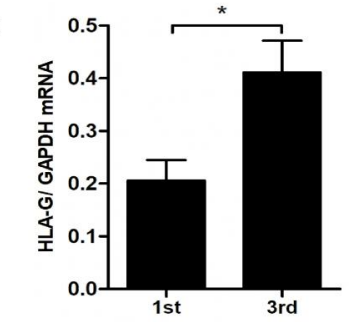

E

$\mathrm{F}$
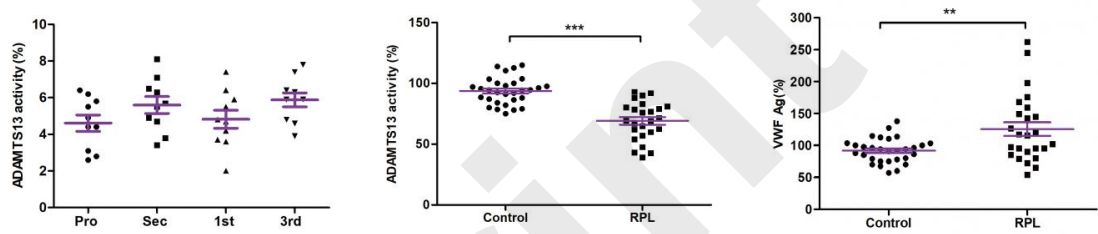

ADAMTS13 activity was detected in the concentrated conditioned media of human endometrium throughout the menstrual cycle and pregnancy. 

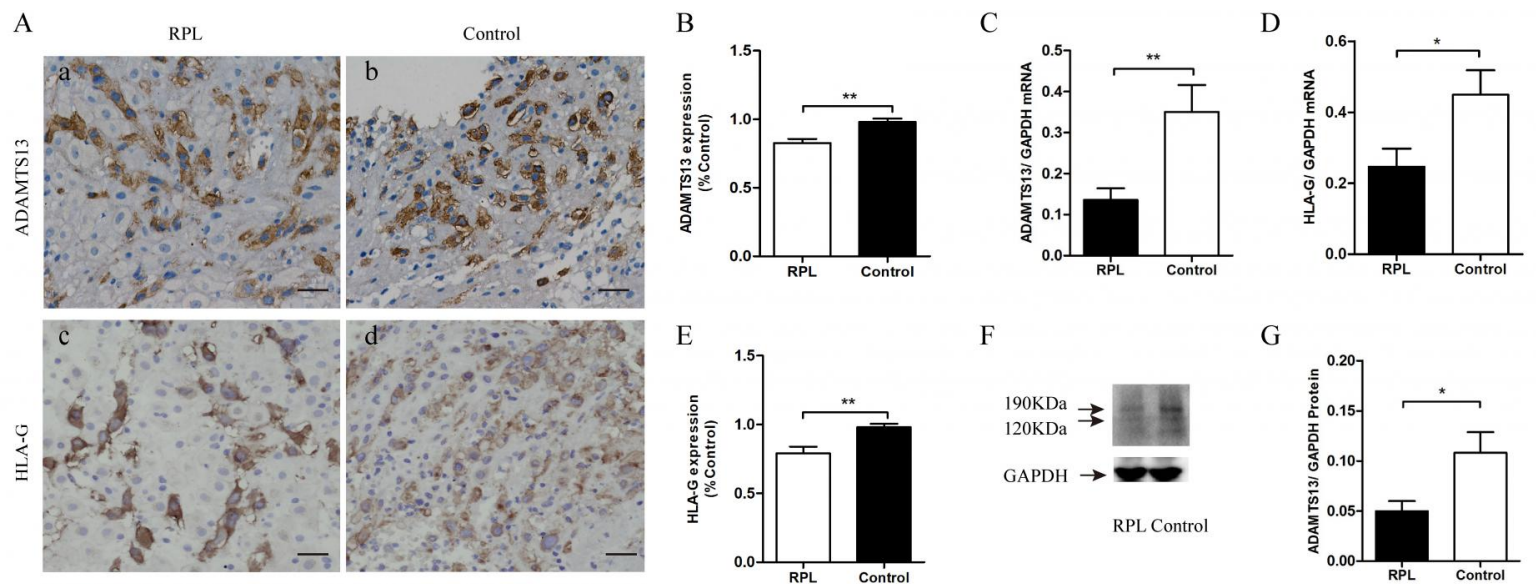

G
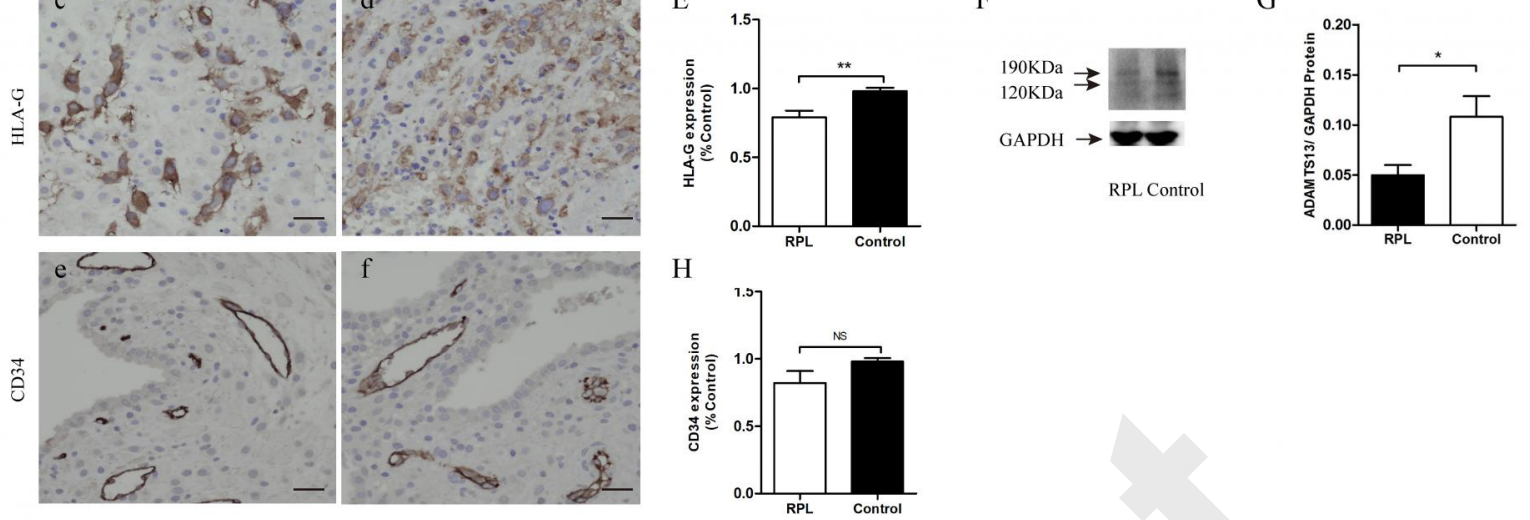

ADAMTS13 expression is down-regulated in RPL decidua. 